\title{
LA RENOVACIÓN DE LAS POLÍTICAS EDUCATIVAS PARA LA PRIMERA INFANCIA
}

\author{
Renewal of education policies for early childhood \\ Renovação das políticas de educação para a infância
}

\author{
Ana de Castro Calvo \\ Florida Universitària, España. Fono: +34 656990125. Correo electrónico: \\ acastro@florida-uni.es \\ Jaume Martínez Bonafé \\ Universitat de València, España. Fono: +34 686209760. Correo electrónico: \\ jaume.martinez@uv.es
}

\begin{abstract}
Resumen
El presente artículo se enfrenta a la situación de las escuelas infantiles en el Estado Español, a través de diferentes ejes de análisis: el concepto guardería vs escuela infantil; el currículum, los tiempos y los espacios; la fragmentación en ciclos de la identidad de etapa; la titularidad privada versus pública; la formación del profesorado y las políticas del municipalismo. Defendiendo posteriormente un modelo de escuela infantil para la vida al servicio de la ciudadanía responsable. Con ello pretendemos poner en primer plano del debate educativo a la primera infancia, las políticas educativas que la regulan y las prácticas de la vida cotidiana en las escuelas infantiles educativas, fundamentadas en el compromiso docente con las pedagogías críticas y renovadoras.

Palabras clave: educación infantil, renovación pedagógica, política educativa, infancia.

Abstract

The present article is facing a situation of the nursery schools in the Spanish State, through different axs of analysis: the concept nursery vs infant school; the curriculum, the times and spaces; fragmentation in cycles of the identity of stage; private ownership versus public; the training of teachers and the policies of the municipality. Defending


subsequently a model of infant school for life at the service of responsible citizenship. With this we want to put in the foreground of the educational debate on early childhood, educational policies that govern them and the practices of everyday life in the nursery schools educational, rooted in the teacher commitment with the critical pedagogies and renovating.

Keywords: infant education, pedagogical renewal, educational policy, children.

\section{Resumo}

Este artigo está enfrentando a situação das escolas infantis no estado espanhol, através de diferentes linhas de análise: o conceito de berçáriovs escola infantis; o currículo, os tempos e os lugares; fragmentação da identidade dos ciclos do estágio; privada versus propriedade pública; a formação do corpo docente e a política do municipalismo. Então a defender um modelo de crianças em idade escolar para a vida a serviço da cidadania responsável. Com isso temos a intenção de colocar em primeiro plano do debate educação para a primeira infância, políticas educacionais que regem e as práticas da vida cotidiana em escolas de educação infantil, baseado no compromisso de ensino de pedagogias críticas e inovadoras.

Palavras chave: educação infantil, renovação educação, a política de educação, criança.

\section{El punto de partida}

A pesar de que existe un consolidado discurso en el ámbito académico y profesional, sobre la transcendente relevancia educativa y social de la etapa 0-6 años, siguen primando en las políticas educativas para la primera infancia, en las prácticas profesionales y en las creencias familiares, un discutible elenco de cuestiones a las que este artículo, además de someter a discusión, pretende responder con propuestas de renovación pedagógica. Veamos algunas de las que aquí consideraremos más discutibles, y sobre las que construiremos respuestas de renovación en el marco de las políticas educativas.

\subsubsection{La escuela infantil versus "guardería" o servicio asistencial y de cuidado}

Hasta hace pocas décadas, en el Estado Español, las políticas de escolarización de los niños y niñas entre los 0 y los 6 años eran escasas, reducidas a una oferta en 
"preescolar" de los 4 a los 6 años, y entre los 0 y los 4 en "guarderías" con un carácter exclusivamente asistencial. La gran mayoría de las "guarderías" destinadas a sustituir a los padres y madres que acuden a sus jornadas laborales, no requerían del marco jurídico y pedagógico exigible a una escuela infantil: un proyecto pedagógico que responda a las necesidades educativas de los niños y a las niñas de este tramo de edad, pero también otros requisitos respecto a sus instalaciones, equipamiento o personal que participa en el mismo. La preocupación familiar durante esas horas queda subsanada con una agenda escolar que hace las veces de relato de la maestra con aquello que los padres deben saber que ha ocurrido durante las ocho horas a sus hijos e hijas: a) si ha hecho caca; b) si ha desayunado, comido y merendado todo; y, c) si ha dormido la siesta. Antes que un proyecto educativo, aquí la atención se centra en el ocio y cuidado de los niños y niñas.

La educación -infantil- se opone a esa concepción miope de guarda y custodia de los niños y los niñas. Educar supone "la sabiduría de conducir fuera, de manera equilibrada y sin falsas precocidades, las capacidades de los niños y las niñas desde el nacimiento" (Alcrudo, et.al. 2015:38). En contraposición guardar en guardarías parte de una imagen de infancia en la que debemos auxiliar al pobre desvalido en sus necesidades básicas; y, por otra parte, auxiliar a una mente vacía la cual debemos ir rellenando, dejando a la infancia sin derechos e ignorando sus capacidades.

A esta empobrecida concepción de las guarderías, se suma también la empobrecida concepción sobre el segundo ciclo de Educación Infantil (3-6 años), donde hay un gran afán propedéutico por adelantar contenidos y metodologías que preparen a los niños y a las niñas para la etapa educativa posterior, la Primaria.

\subsubsection{Currículum, tiempos y espacios}

Cuando la oferta en el primer ciclo 0-3 años se sitúa en el marco de las "guarderías infantiles", estas acciones ocurren habitualmente en plantas bajas de edificios de viviendas, con escasa luz solar, patios exteriores de hormigón que corresponden a patios de luces, y una gran agresividad polisensorial en lo que hace referencia a los colores de las paredes, los mobiliarios o los materiales de plástico con luces y colores estridentes de objetos supuestamente didácticos. Recordemos, sin embargo, algunos de los requisitos que las instalaciones deben cumplir si quiere ser autorizada como "escuela 
infantil": el centro debe ubicarse en locales de uso exclusivamente educativo; cada clase o unidad debe tener su propia aula de aprendizaje, que tendrá una superficie de dos metros cuadrados por cada niño y en ningún caso debe ser menor de 30 metros cuadrados; las aulas que estén destinadas a niños menores de 2 años deben contar con un área específica para el descanso de los menores y otra para la higiene; el centro tiene que disponer de una sala de usos múltiples con una superficie entre 30 y 40 metros cuadrados; y los niños deben tener a su disposición un patio de juegos exclusivo del centro con una superficie mínima de 60 a 75 metros cuadrados; finalmente, se debe contar con un espacio adecuado para la preparación de alimentos, así como con aseos destinados al personal del centro separados de los de los niños.

En el caso de la oferta de "preescolar" (3-6 años) la disposición arquitectónica tiene que ver más con el enfoque propedéutico de escolarización temprana, que con las necesidades e intereses infantiles. Se le añaden sillas y mesas -con un poco de suerte circulares, cuando no, individuales- para preparar a los niños y a las niñas con una "buena" base de lecto-escritura y cálculo antes de su paso a la etapa escolar. Habitualmente se les somete a intensivas horas sentados frente a un DIN A-4 con actividades desvinculadas del deseo y la experiencia del infante. Este segundo ciclo se instala en la mayoría de los casos en edificios de Educación Primaria, sin patio propio, ni cumplimiento de los metros cuadrados que deben tener las aulas, sin las adaptaciones requeridas para la primera infancia.

Necesitamos abrir un diálogo entre arquitectura y pedagogía para los edificios destinados a escuelas infantiles aporten "experiencias ricas, de percepciones sensibles, de espacios vinculados emocionalmente y que permitan una apropiación de los lugares mediante el juego" (Cabanillas, Eslava y Tejada, 2005:59). Se trataría también de:

“ (...) huir del infantilizar los espacios, que siempre son de niños para niños, pero pensando en el sentido de que no saben hacer nada, y decorar con Mickey y cosas así; y no, este es un espacio donde tiene cabida cualquier cosa y ponen cosas como letras y números por las aulas, en vez de poner los dibujos que han hecho ellos, o que todo esté colocado a su nivel” (De Castro, 2015:245).

La metodología que se lleva a cabo, por tanto, en muchos casos, son programaciones rígidas de fichas, perdiendo la esencia de la globalización, el aprendizaje holístico, la educación activa y flexible. Todo ello en detrimento del juego, 
la psicomotricidad, la música, la manipulación y la experimentación; ya desde el primer ciclo de Educación Infantil, con lo cual se pierde el sentido radical del concepto Educación Infantil:

"Cuando la educación infantil tiene poco de infantil, las experiencias educativas resultan mucho menos interesantes y estimulantes, pudiendo incluso generar dificultades en los alumnos, y en todo caso, no sacar de ellos las potencialidades que poseen" (Paniagua y Palacios, 2005:14).

Este tipo de metodología se traduce en una organización del tiempo escolar con distintos y diferenciados momentos, por un lado, la jornada académica, y por otro lado los momentos básicos para los rituales cotidianos: el aseo personal, el desayuno, la comida, la siesta o la bienvenida/ despedida. Dividiendo así el horario en momentos educativos y momentos no educativos. Cuando desde una concepción de escuela infantil todos los momentos que tienen lugar durante la jornada escolar son momentos educativos.

\subsubsection{Fragmentación en ciclos de la identidad de etapa}

Aunque en el contexto del Estado Español, la importancia de este período se intentó plasmar legislativamente en la Ley General de Educación de 1970, el desarrollo no se correspondía con las expectativas generadas y las reivindicaciones de los distintos movimientos educativos que, en aquel momento, defendían la ampliación de la Educación Infantil desde los cero años hasta los siete e incluso los ocho años como un derecho desde el nacimiento, y una formación inicial y permanente de maestros y maestras adecuada a esta etapa. Esta misma ley, reconoció la educación preescolar como un nivel educativo, pero sin incluirlo en el sistema general, por tanto tenía carácter voluntario. Se dividía en dos etapas. La primera, Jardín de Infancia, para niños y niñas de dos y tres años y tendrá un carácter similar a la vida del hogar; y la segunda, la Escuela de párvulos, para niños y niñas de cuatro a cinco años, la cual promoverá las virtualidades del niño y la niña.

No será hasta 1990 con la LOGSE (Ley Orgánica General del Sistema Educativo), hasta que aparece el concepto de Educación Infantil como tal y se incluye en el sistema educativo y se reconoce la identidad de etapa 0-6 años con carácter educativo, reconociendo por primera vez el transcendente valor educativo de esta 
primera etapa de la escolarización. Dividida en dos ciclos: el primero de los cero a los tres años, y el segundo de los tres a los seis.

Sin terminar de aplicar la LOGSE, el gobierno conservador promulgó la LOCE (Ley Orgánica de Calidad Educativa) en el año 2002 que vuelve a dejar fuera del sistema educativo el primer ciclo de 0-3 años dándole nuevamente un carácter educativo asistencial. Posteriormente, en el año 2006, la LOE (Ley Orgánica de Educación) vuelve a recuperar la identidad de la etapa 0-6 años con la finalidad de contribuir al desarrollo físico, afectivo, social e intelectual de los niños y las niñas.

En el borrador de la última propuesta legislativa educativa, la LOMCE (Ley Orgánica para la Mejora de la Calidad Educativa) de 2013, no se propone ningún cambio en la etapa de Educación Infantil. Confirma los principios establecidos por la LOE, mantiene su identidad educativa, su gratuidad y su voluntariedad. Aunque cabría que el entonces Ministro de Educación Wert en la Comisión de Educación del Congreso de los Diputados el 31 de enero de 2012 afirmó que: “...la cuestión de la educación de 0 a 3 años (...) yo creo que eso no es educación, que es básicamente conciliación” (Alcrudo et.al., 2015:113).

En cualquier caso, la ambigüedad con que ha venido considerándose el tramo 03 años ha fomentado y mantenido una discutible fragmentación en dos tramos, con atenciones y servicios diferenciados. Sin embargo, es sabido, por experiencias y modelos de otros países (el caso de Reggio Emilia es emblemático) de las indiscutibles ventajas de la continuidad en un mismo centro y el tratamiento unitario y global de etapa para un tramo único 0-6 años. En la investigación de José Domínguez, se señala:

\footnotetext{
"Todos los participantes/colaboradores en la investigación cualitativa coinciden en dos aspectos relevantes sobre este tema: a) La Educación Infantil debe ser una etapa educativa única y unitaria. b) La Educación Infantil no se ha consolidado como etapa educativa unitaria en el sistema educativo español, s pesar de los 40 años de lucha a favor e esa unidad el movimiento en defensa del carácter esencialmente educativo del periodo 0-6 años" (Domínguez, 2012:64)
}

\subsubsection{Privada versus pública. Equidad desde el nacimiento}

En contextos con políticas educativas conservadoras, el incremento de los procesos de privatización fue en aumento (Carrera y Luque, 2016). En el caso de ciudades como 
Madrid o Valencia (España), ta mayoría de las escuelas infantiles, han pasado a estar gestionadas por empresas privadas que no siempre atienden a criterios educativos de calidad, democracia, transparencia y contratación del profesorado cualificado. El desvío de fondos públicos al mantenimiento de centros privados mediante fórmulas como el cheque escolar, convenios o similares cada vez son más frecuentes.

La falta de una red potente de escuelas infantiles públicas supone un ataque importante a la igualdad de oportunidades e impide la conciliación familiar y laboral de hombres y mujeres. El alumnado que procede de sectores más desfavorecidos no tiene acceso en los primeros años de vida a escuela infantiles públicas de primer ciclo.

Bajo este paraguas, las escuelas infantiles privadas, no se cuestionan el discurso de la equidad como algo importante o a tener en cuenta -si es que se lo cuestionan- si no que viven las escuelas infantiles y la primera infancia como una empresa y una fuente de ingresos, $\mathrm{y}$, por tanto, la rendición de cuentas es a los clientes -los padres y las madres- y dando una "buena imagen" al barrio para seguir creciendo en volumen de negocio.

Esta bidireccionalidad, ha venido ligada habitualmente -aunque no podemos generalizar- al concepto de escuela tradicional como escuela privada o escuela moderna como escuela pública. La escuela infantil privada, en la gran mayoría de las ocasiones ha pertenecido y pertenece a las clases más privilegiadas, en cambio, la escasa oferta de la escuela infantil pública, ha estado y está destinada a las clases populares. Esta situación hace que todos y todas no tengamos las mismas oportunidades desde el nacimiento, y que, por tanto, el sistema educativo no cumpla con su función de equidad reconociendo a las personas sin distinción, para que todos los niños y las niñas de cualquier grupo social reciban una educación asegurando los mismos estándares de calidad. Velando para que el sistema educativo o las escuelas infantiles no separan a las personas o grupos.

\subsubsection{Formación de profesionales:}

Es obvio que una etapa educativa tan relevante requiere de profesionales de la educación preparados, con competencias y estilo educativo específico para esta etapa. Sin embargo, hasta las últimas reformas de los planes de estudio del Magisterio no se contempló un título propio de Grado de Maestra/o de Educación Infantil. A esto 
debemos añadir el peso de una tradición escolar que sitúa a la Educación Infantil como un "preescolar" que, como ya se comentó, muestra un fuerte sesgo en métodos y contenidos orientados a preparar para los aprendizajes instrumentales de la Educación Primaria. La escasa formación inicial y el considerable peso de la escolarización, en la que se incluye "el recuerdo" de los modelos pedagógicos vividos por los maestros y maestras durante sus años de escolarización, enmarcan a la Educación Infantil en las pedagogías más tradicionales. Este tipo de situaciones resalta el individualismo por encima de una concepción colectiva, un cuerpo único de docentes que debata, cuestione y analice tanto la concepción social y pedagógica del puesto de trabajo como la formación inicial y permanente de educadores y educadoras, y maestras y maestros. Dejando de lado la investigación, la puesta en común y el análisis colectivo.

La orientación de los planes de estudio en las Facultades de Educación, primando las didácticas específicas sobre una formación de fuerte raíz integral pedagógica, tampoco facilita la adquisición de saberes de renovación pedagógica que, además de hacer frente el peso de la tradición contemplen la profesión como un compromiso ciudadano y al docente como un o una intelectual, como modelo no sólo de pedagogía en el aula, sino también de ciudadanía, ciudadanía que ha de ser capaz de cultivarse en la escuela. Como señala Philippe Meirieu:

"Formar a los docentes para una escuela democrática es preparar a los hombres y a las mujeres en dos aspectos vinculados consustancialmente: un oficio de experto y un compromiso de ciudadano; y no hay que sacrificar el compromiso ciudadano en beneficio del oficio de experto" (Meirieu, 2006:130).

Desde este enfoque estaríamos ayudando a los y las docentes de Educación Infantil -o cualquier etapa del sistema educativo- a desarrollar un perfil profesional crítico, reflexivo e investigativo de su propia práctica. Como se ha venido haciendo en el campo de la innovación educativa y la renovación pedagógica que, gracias al esfuerzo y la creatividad investigadora de maestras y maestros de Educación Infantil, se han ido constituyendo referentes importantes para la formación de otros profesionales.

Por otra parte, se mantiene una doble y diferenciada titulación entre los y las técnicos y maestros y maestras de Educación Infantil en condiciones laborales, salariales y curriculares muy desiguales. Esto supone desigualdad en lo que respecta a la formación académica de los y las docentes del segundo ciclo de Educación Infantil (3-6 
años), ya que los técnicos tienen una mayor formación práctica en sus estudios iniciales; mientras que los maestros y las maestras que estudian un grado universitario tienen una gran parte del plan de estudios destinada a la formación teórica de las diferentes áreas didácticas.

\subsubsection{Municipalismo}

El derecho universal a la educación desde los cero años exige procesos de implicación familiar y ciudadana potentes y autogestionados, en los que el territorio, el barrio, el municipio cobra, en el caso de la Educación Infantil, un protagonismo especial. Así lo entendieron los movimientos sociales y vecinales en las postrimerías del franquismo, promoviendo en la mayoría de las ciudades españolas escuelas infantiles cooperativas con proyectos educativos renovadores. Esta oportunidad de municipalismo y autogestión, en el marco de políticas públicas a las que no acudía el Estado, fue perdiendo fuerza durante la democracia, en los sucesivos gobiernos tanto de la socialdemocracia como de la derecha conservadora. Lo que se inicia como un proceso de desmantelamiento de la participación ciudadana abre la puerta a posteriores privatizaciones y externalizaciones de servicios públicos que asumían o podrían asumir los municipios, como es el caso de las escuelas infantiles municipales.

Se frustró así la oportunidad de desarrollar políticas educativas locales que nacieran del cooperativismo y el asociacionismo, las cuales se adaptan mejor a las realidades concretas vinculando la escuela a otras dimensiones de la vida colectiva de los pueblos y los barrios. Esta participación municipal, que abría las escuelas infantiles a los movimientos del barrio y a la participación de las familias fue usurpado por políticas educativas neoliberales que franquician las escuelas infantiles 0-3 años, desintegrando, dispersando y deteriorando la vida y el sentido profundo de este tipo de escuelas.

\section{Un camino distinto: una escuela infantil educativa.}

La presión de las políticas neoliberales y sus consiguientes recortes en el gasto público han venido provocando respuestas populares importantes (Carrera y Luque, 2016). En educación, los últimos años de gobierno de la derecha han obtenido la respuesta de potentes movimientos vecinales, plataformas, "mareas", coordinadoras, que además de una presencia crítica importante en la calle, generaron estudios bien informados. En 
educación infantil es paradigmático el informe La Educación Infantil de 0-6 años en España, promovido por la Plataforma Estatal en defensa del 0-6. (Alcrudo, et.al. 2015).

Tales movimientos e informes recuperan lo mejor de las pedagogías críticas y de la potente tradición de los Movimientos de Renovación Pedagógica, para defender otro camino para la escuela infantil, en el que la educación basada en un profundo sentido de la libertad, la justicia y la democracia participativa, ponga el crecimiento emancipado del ser humano en el punto de mira de sus pedagogías.

Frente a la tradicional guardería o escuela asistencial se defienden aquí escuelas infantiles integrales y democráticas, que salgan al mundo real, saltando los muros de una escolarización escolástica y caduca (Malaguzzi, 2001) para dejar que los flujos entren de dentro hacia fuera y de fuera hacia dentro, para que la comunidad se constituya permeable, transparente y creativa.

Estamos hablando por tanto de una escuela infantil para la vida, creada y pensada para los niños y las niñas desde los cero años, que acoja toda su vida en su singularidad, con las necesidades de todos y cada uno. Una escuela infantil que invite a descubrir la vida, el mundo, las cosas, los objetos; y que en este descubrimiento se aprenda, se pregunte, se comunique, se haga, se toque, se huela, se manipule, etc. Una escuela que ponga en relación los sentidos, la experiencia social y cultural, las biografías y vida cotidiana, y el derecho de todo ser humano a explorar las enormes posibilidades de su crecimiento integral en libertad.

Esta propuesta pone de manifiesto la pedagogía de la experiencia basada en el propio descubrimiento. Como decía John Dewey (1967) reconocer y compartir con los niños y niñas experiencias de primera mano, que partan de sus propias necesidades, intereses y curiosidades. Para que se creen las condiciones en las que los niños y las niñas lleguen a sus propios descubrimientos, y esos descubrimientos signifiquen sus aprendizajes. Los aprendizajes llegan solos cuando se sienten queridos, se sienten seguros, se sienten mirados, se sienten acogidos, cuando se respetan sus rabietas, sus llantos, sus tristezas y sus alegrías. Esta idea es básica en todas las edades, pero, como bien argumenta Emmi Pikler (2012) en esta etapa infantil es más necesaria.

Esta mirada de escuela infantil, necesita con urgencia dos cambios legislativos, culturales y pedagógicos: por un lado, la reformulación de la formación inicial y 
permanente de los maestros y maestras tanto del primer como del segundo ciclo, y por otro lado, la revisión de los curricula que nos proporcionan las administraciones educativas.

\subsection{Sobre la formación inicial y permanente de los y las docentes}

En primer lugar, debemos enfrentarnos con la escasa formación académica que existe para los y las educadoras del primer ciclo de Educación Infantil (0-3 años), a través de un Ciclo Formativo de Grado Superior en Educación Infantil de dos años de duración. Esto supone una gran desigualdad con respecto a la formación académica de Grado de los y las docentes del segundo ciclo de Educación Infantil (3-6 años), un título universitario de cuatro años de duración. Por eso, desde este espacio, creemos necesario poner de manifiesto la revisión y equiparación de titulaciones, condiciones laborales y currículum de formación de los y las técnicos superiores de Educación Infantil, con los maestros y maestras de Educación Infantil.

Alfredo Hoyuelos (2011) añade que en la formación inicial de los maestros y las maestras de Educación Infantil 0-6 años, se debería trabajar sobre cinco planos complementarios que desvelan un tipo de perfil profesional:

a. El plano relacional vincular: se debe dar desde las facultades una formación que acoja el lado emocional de cada uno de nuestros alumnos y alumnas para que posteriormente puedan construir lazos de seguridad, empatía y confianza con sus alumnos y alumnas. Y a su vez, sean profesionales capaces de comprender la expresión emocional del alumnado.

b. El plano sociopolítico: los y las docentes -sea cual sea el nivel en el que se muevan- no pueden comprender su profesión únicamente desde un punto de vista didáctico, sino también desde el punto de vista de posibilitar la transformación social.

c. El plano relacional y cultural: una cultura que permita a los y las profesionales los matices que los niños y las niñas tienen para comprender el mundo al que tratan de dar sentido. Considerando así que los profesionales de cada escuela sean los que elaboren sus propios proyectos de trabajo transdisciplinarmente. 
d. El plano de investigación: la profesional o el profesional como investigador e investigadora permanente. Investigación como búsqueda permanente del desarrollo de la labor profesional, dejando huella documental y narrativa de las propias prácticas educativas.

e. El plano de diseño de escenarios estéticos: concibiendo al docente no sólo como alguien que enseña, sino como mediador que organiza y coordina propuestas, y que acepta las invitaciones que le llegan de los niños y las niñas. En este sentido, los docentes -con sabiduría dialógica- deberían conocer las formas de proponer experiencias que enganchen, respetando sus ritmos y haciéndoles dueños subjetivos de sus propios ritmos de aprendizaje.

Estos cinco planos para el desarrollo profesional, se podrían cultivar mediante la documentación narrativa de las experiencias pedagógicas. Abogamos por este tipo de metodología, para trabajar tanto desde la formación inicial del profesional, como desde el puesto de trabajo, los y las docentes podrían indagar, reconstruir y hacer públicos sus saberes y prácticas pedagógicas que van teniendo lugar en sus experiencias escolares.

De esta manera, podríamos estar dotando al cuerpo único de docentes, de herramientas conceptuales y metodológicas para su desarrollo profesional.

Este tipo de formación inicial, debería continuar a lo largo de la vida laboral, pudiendo encontrar espacios de formación permanente que permitiese a los profesionales continuar formándose desde la experiencia y las prácticas de los otros y las otras, desde la horizontalidad, los intereses comunes, las buenas prácticas y la elaboración de un currículum basado en la investigación y colaboración entre docentes, y en consecuencia, que estos saberes pudieran retornas a sus aulas como vía para la formación permanente con estrategias de sistematización de la práctica como los Círculos de Cultura freirianos, la La Investigación/Acción Participante, La Documentación Narrativa de Experiencias o La Documentación Pedagógica (Martínez, 2006, 2011).

Con este cuerpo único de enseñantes, que abarque desde la Educación Infantil hasta la Universidad, se estaría rompiendo con la visión social de inferioridad con que se ha tratado institucionalmente al profesor o profesora de Educación Infantil. Así permitiríamos la circulación del profesorado a través de ciclos y niveles cultivando 
herramientas conceptuales y procedimentales desde y para los distintos niveles del sistema educativo.

Otra estrategia, que se podría llevar a cabo y que también se defiende desde la propuesta pedagógica de Loris Malaguzzi y la experiencia de Reggio Emilia, es la apertura del puesto de trabajo a una estructura de pareja educativa. Enriqueceríamos así los roles de los y las docentes, mejorando su práctica, diluyendo sus angustias y enriqueciendo las pequeñas acciones de la vida cotidiana. Se trata de una complementariedad en la que se gana en autonomía y libertad didáctica desde la confrontación y el diálogo. Compartiendo la horizontalidad pedagógica, la pedagogía de la escucha -no sólo hacía los niños y las niñas, sino también hacia los compañeros y compañeras-, y la democratización pedagógica.

\subsection{El currículum en Educación Infantil}

Como hemos señala en la primera parte del artículo, las leyes que se han ido sucediendo desde la dictadura, han promovido modelos educativos que centran su concreción curricular para Educación Infantil en un enorme abanico de contenidos educativos. Contenidos completamente alejados de las realidades, intereses, biografías y deseos de los niños y las niñas.

Este abanico de contenidos se materializa en tres áreas de conocimiento: conocimiento de sí mismo y autonomía personal, conocimiento del entorno y lenguajes: comunicación y representación (Para el primer ciclo, en la ciudad de Valencia: Decreto 37/2008, de 28 de marzo, y Decreto 2/2009, de 9 de enero. Para el segundo ciclo: Real Decreto 1630/2006, de 29 de diciembre). Cada comunidad autónoma del estado español tiene competencias plenas en educación, produciéndose algunas variaciones en las propuestas curriculares de unas a otras.

Es por ello, por lo que deberíamos tener presente no tanto los contenidos educativos a trabajar, sino asegurar que el currículum de Educación Infantil, como se cita en el diseño curricular de Educación Infantil que editó la Generalitat Valenciana en el año1990, cumple con diversas funciones: función compensadora e integradora; función de socialización; función de individualización, adquisición de los aprendizajes básicos; igualdad de oportunidades; justicia social; convivencia y conocimiento de las realidades y modos de organización político-social cercanas. 
Por tanto, el currículum que se establezca partiendo de estas funciones, debe ser un currículum en permanente desarrollo, revisión y actualización, para garantizar que la propuesta de diseño curricular que realice cada realidad, se ajusta a su contexto.

Para poder poner en marcha todo lo mencionado anteriormente, necesitamos de metodologías que adopten un enfoque globalizador. Este enfoque globalizador puede materializarse en Educación Infantil, a través de metodologías como los centros de interés, los proyectos de trabajo, la investigación del medio, el trabajo por ambientes de aprendizaje, los talleres, la psicomotricidad vivenciada o el juego heurístico. Metodologías que combinan los lenguajes y las inteligencias no verbales de los niños y las niñas para adquirir nuevas modalidades que les enriquezcan, que les permita encontrarse consigo mismo y con los demás.

Un planteamiento metodológico que cree lugares de crecimiento donde los niños y las niñas tienen la posibilidad de hacer cosas y a la vez, reflexionar sobre lo que están haciendo. En el que es posible curiosear, probar, errar, concentrarse, buscar alternativas, soluciones, estar en calma, explorar, ponerse en acción, construir, etc., para que puedan enriquecerse a nivel intelectual, afectivo y relacional; "un lugar de investigación en el que la imaginación, el rigor, el experimento, la creatividad y la expresión se entrelazarían y se complementarían mutuamente" (Dahlberg y Moss, 2013:45).

Este enfoque supone una nueva manera de concebir la relación conocimientosujeto, poner el conocimiento al servicio de los niños y las niñas, y no a éstos al servicio del conocimiento. Para ello, se deben desarrollar situaciones de aprendizaje a través de un currículo integrado que respete los conocimientos previos, las necesidades, los intereses, los deseos, los ritmos y las biografías de cada niño o niña; definiéndose como un proceso de construcción colectiva, complejo, dinámico y en permanente investigación, para que genere la articulación entre los desarrollos cognitivos, afectivos, sociales y las demandas de aprendizaje de los niños y las niñas con el conocimiento y el desarrollo de las "herramientas para la vida".

Por todo ello, la planificación curricular del currículum de Educación Infantil debe contemplar todas las acciones planteadas para que los niños y las niñas desde el nacimiento, se enfrenten a contenidos y objetivos que sean funcionales, prácticos y 
significativos sin que ello quite veracidad a la diversidad y complejidad de elementos que forman el entorno en que se desarrollan.

2.3. La organización de la escuela, la participación de las familias y el municipalismo

Sería un modelo organizativo inspirado en Célestin Freinet cuando sugiere la cooperación educativa, en el que los y las docentes aprenden a tomar decisiones colectivamente apoyados en la cooperación entre iguales y la cultura del apoyo mutuo. En ese marco conceptual, la participación del profesorado implicaba igualmente la participación entre iguales del alumnado y las familias. Las decisiones trascienden las cuestiones inmediatas, los problemas puntuales, las necesidades concretas de recursos $\mathrm{u}$ orientaciones. Abriendo una práctica reflexiva que pone en cuestión el propio modelo de escuela y, por tanto, el sentido de la escolarización en las sociedades actuales. Esta es la cuestión de raíz, el compromiso con la mejora permanente de la escuela que es también el compromiso con la transformación social. Ese es el verdadero reto de la participación, dentro y fuera de la escuela (Martínez, 2006:26).

El papel que tiene la familia los primeros años de vida de los niños y las niñas, es incuestionable; esta conexión ayuda a la consecución de un proyecto educativo colectivo, que ayuda a fortalecer contactos afectivo-emocionales, y a participar en la introducción del mundo social y cultural en el que viven los niños y las niñas. La relación que se establecen entre ambos contextos de vida debe partir de los intereses, de los bagajes familiares y de las culturas de los niños y las niñas. Este camino de interacción, supone respetar maneras de hacer y pensar, y reconocerse positivamente a unos y a otros. Este "estar" va más allá de interacciones u observaciones que tienen lugar a la entrada y a la salida de la escuela -de manera informal-, sino que hay que esforzarse por cambiar el rol y crear relaciones estables entre el personal de la escuela y las familias. La presencia conjunta de ambos contextos hace que sea de mayor calidad y más eficaz la tarea educativa.

"Participación de la familia en la educación, entendida como la posibilidad de incidir, de decidir, de opinar, de aportar, de disentir y de actuar en diversos campos de la educación, acordados previamente y de común acuerdo entre docentes, padres y otros agentes educativos, con funciones definidas y comprendidas por ambos. En la medida que la preocupación por participar es el niño concebido en su integralidad y como sujeto de 
derechos, puede implicar actuar tanto en el campo educativo, como de la salud, del trabajo, de la mujer u otro, siempre que tenga relación con el mejoramiento de las condiciones de vida de los niños y niñas para mejorar sus aprendizajes y desarrollo" (Blanco y Umayahara, 2004:39).

Pero estos procesos participativos estarían incompletos sin considerar las políticas públicas municipales, la estructura barrial de la ciudad y los movimientos sociales que se vienen configurando con nuevas formas de acción política (Della Porta, 2011). Los barrios y los pueblos deberían ser tejidos sociales activos en la toma de decisiones, apostando por un cambio en el paradigma de las sociedades futuras desde otra concepción de lo público y del papel de la educación infantil pública, viviendo la escuela infantil como un lugar de encuentro, de interacción y conexión entre las personas de la comunidad. Ejemplos potentes de municipalismo, como los de Reggio Emilia donde, como dice Carla Rinaldi (2011) abogan además por conseguir el potencial emancipador de la democracia, ofreciendo a cada niño y cada niña la posibilidad de sentirse ciudadano activo, dentro del seno de una comunidad democrática. O en el marco de las ciudades educadoras en Brasil, fomentando una política activa lo es barrios para la creación de las escuelas infantiles (Morigi, 2016). O como ocurrió en la ciudad de Valencia en los años 70 con la creación del Patronato de Escuelas Infantiles.

\subsection{La integración de la estética, la ética y la política}

Lo señalado hasta ahora, nos lleva a otra dimensión, más holística y compleja que necesita poner en relación todas las dimensiones anteriores. Estaríamos hablando de la integración de la estética, la ética y la política en todos los proyectos educativos de las escuelas infantiles públicas. Con la integración de estos tres elementos interdependientes, dotaríamos a las escuelas de sentido vital y cultura en cada uno de los momentos del día a día de la pedagogía de la infancia. Alfredo Hoyuelos (2001), en su tesis doctoral, hace un gran esfuerzo por concretar la obra pedagógica de Loris Malaguzzi a través de estos tres elementos, y dice, en su libro "La ética en el pensamiento y obra pedagógica de Loris Malaguzzi" que la integración de la estética, la ética y la política, supone:

"Un proyecto que, narrado y testimoniado sin retóricas, reconoce a los niños y niñas recursos y potencialidades inéditos, que desvela las riquezas insospechadas de la especie 
humana, que trasciende -como expresa el arte- las culturas individuales. La pedagogía, el pensamiento y la obra de Loris constituyen una pedagogía ética, estética, política, compleja, biológica, cultural, relacional, sistémica, participante, transgresora y constructiva que indaga y narra -como proyecto inacabado- una imagen de una infancia con derechos universales. En ese sentido, el pensamiento y obra pedagógica de Loris Malaguzzi y de los ciudadanos (niños y adultos juntos) de Reggio Emilia son exponente del desarrollo de un dialógico proyecto humano (y no sólo escolar) de escucha de las esperanzas futuras de los hombres, más allá de las culturas particulares" (Hoyuelos, 2004:26).

Además, bajo este paradigma de escuela infantil, la educación debe estar al servicio de una ciudadanía responsable y consciente de su capacidad transformadora, y especialmente de empoderar aquellos sectores sociales y culturales más necesitados. Para ello, las políticas públicas deben concretarse en proyectos educativos que garanticen una educación para el bienestar individual y colectivo, y para el desarrollo integral y sostenible de los pueblos. Es necesario crear un espacio ciudadano que pueda ayudar, contribuir y provocar una crisis en el modo de pensar y un conflicto sobre el significado de la escuela, con el único objetivo de crear crisis de pensamiento. Crisis que puede abrir nuevas posibilidades y expectativas, preguntas y soluciones alternativas, oportunidades a la interpretación y maneras de ver las cosas. Sin este cambio de paradigma, se ven constantemente truncadas las iniciativas pedagógicas de transformación social. No partimos de cero. En el Estado Español existen experiencias muy potentes en diferentes municipios (Pamplona, Barcelona, Valencia, etc.) y es un referente de primer orden el histórico proyecto de Reggio Emilia en Italia.

Pensad -decía Malaguzzi, 2001- que todos los que en estos últimos cuarenta años se han puesto, seriamente, a investigar sobres los niños y las niñas han terminado siempre por descubrir no tanto las debilidades y los límites, cuanto la fuerza sorprendente y extraordinaria de sus potencialidades. Pero pensad, también, que esta increíble acreditación de las capacidades de los niños y las niñas desde los cero años no ha modificado para nada las elecciones económicas, políticas y legislativas de los gobiernos nacionales y de los centros de poder internacionales. Y pensad, por tanto, que el destino y los intereses de los niños y las niñas permanecen en los últimos puestos de la escala de prioridades de financiación y de intervención en cualquier país de la Tierra.

Hemos de apostar por un cambio en las políticas educativas locales y poner en marcha centros educativos para la primera infancia donde se reconozca el derecho de 
cada niño y niña a ser sujeto; lugares donde se propone la creación de un ambiente amable para los niños y las niñas, las familias y los maestros y las maestras, dando significado humano, digno y civil a la existencia (Rinaldi, 2011).

El presente artículo propone para las escuelas infantiles 0-3 años y las 0-6 años un nuevo campo de relación en el municipio en el que se enclava, relación que parte de lo colectivo en la toma de decisiones, y que está apostando por un cambio en el paradigma de las sociedades futuras. Es una manera también de apostar por los servicios públicos construidos con y desde la gente, y no desde políticas neoliberales clientelistas. Asumiendo la responsabilidad colectiva y a su vez, creando un proyecto educativo consensuado, compartido y contextualizado. Viviendo la escuela infantil como un lugar de encuentro, de interacción y conexión entre las personas de la comunidad.

\section{Referencias bibliográficas}

Alcrudo, A.; Alonso, A.; Escobar, M.; Hoyuelos, A.; Medina, Á. y Vallejo, A. (2015). La Educación Infantil de 0 a 6 años en España. Madrid: Editorial Fantasía.

Blanco, R. y Umayahara, M. (2004). Participación de las familias en la educación infantil latinoamericana. Santiago de Chile: Oficina Regional de Educación de la UNESCO para América Latina y el Caribe.

Carrera, P. y Luque, E. (2016). Nos quieren más tontos. La escuela según la economía neoliberal. Barcelona: Editorial El viejo Topo.

Dahlberg, G.; Moss, P. y Pence, A. (2005). Más allá de la calidad en educación infantil. Barcelona: Editorial Graó.

De Castro, A. (2015). Renovación Pedagógica y vida cotidiana en el primer ciclo de Educación Infantil. Un estudio de caso. Tesis Doctoral leída en la Universidad de Valencia.

Decreto 2/2009, de 9 de enero, por el que se establecen los requisitos mínimos que deben cumplir los centros que impartan el primer ciclo de Educación Infantil en la Comunidad Valenciana. DOGV 5932.

Decreto 37/2008, de 28 de marzo, del Consell, por el que se establecen los contenidos educativos del primer ciclo de la Educación Infantil en la Comunidad Valenciana. Consellería de Educación. DOGV 5734. 
Della Porta, D. (2011) Lo movimientos sociales. Madrid, CIS/Editoral Complutense.

Dewey, J. (1967). Experiencia y educación. Buenos Aires: Editorial Losada.

Domínguez Rodríguez, José (2012) Educadores y Educandos Infantiles. Una utopía posible. Madrid: Federación de Movimientos de Renovación Pedagógica de Madrid.

Hoyuelos, A. (2004). La ética en el pensamiento y obra pedagógica de Loris Malaguzzi. Barcelona: Editorial Octaedro-Rosa Sensat.

Hoyuelos, A. (2011). Educación Infantil: una canción a varias voces. Revista Tarbiya, Revista de Investigación e Innovación Educativa, 42, 5-11. Universidad Autónoma de Madrid.

Ley 14/1970, de 4 de agosto, General de Educación (LGE).

Ley Orgánica 1/1990, de 3 de Octubre, de Ordenación General del Sistema Educativo (LOGSE).

Ley Orgánica 10/2002, de 23 de diciembre, de Calidad de la Educación (LOCE). Ley Orgánica 2/2006, de 3 de mayo, de Educación (LOE).

Ley Orgánica 8/2013, de 9 de diciembre, para la mejora de la calidad educativa (LOMCE).

Madrid: Editorial Narcea.

Malaguzzi, L. (2001). La educación infantil en Reggio Emilia. Barcelona: Editorial Octaedro- Rosa Sensat.

Martínez, J. (2006). El profesorado antes los discursos y las culturas de participación. En Revista Cuatrimestral del Consejo Escolar del Estado, 3, 23-27.

Martínez, J. (2011) Aprender el oficio docente sistematizando la práctica. En Pérez Gómez, A. (Coord.) (2011). Aprender a enseñar en la práctica: procesos de innovación y práctica de formación en la educación secundaria. Barcelona: Editorial Graó. 
Meirieu, P. (2006). ¿Es posible formar al profesorado para una escuela democrática?. En AAVV, El protagonismo del profesorado: experiencias de aula y propuestas para su formación. Madrid: Ministerio de Educación y Ciencia.

Morigi, V. (2016). Cidades educadoras. Possibilidades de novas políticas públicas para reinventar a democracia. Porto Alegre, Editora Sulina.

Paniagua, Gema y Palacios, Jesús (2005) Educación Infantil. Respuesta educativa a la diversidad. Madrid, Alianza Editorial.

Pikler, E. (2012). Moverse en libertad. Desarrollo de la motricidad global.

Real Decreto 1630/2006, de 29 de diciembre, por el que se establecen las enseñanzas mínimas del segundo ciclo de Educación infantil.

Rinaldi, C. (2011). El diálogo con Reggio Emilia. Escuchar, Investigar, aprender. Perú: Grupo Editorial Norma S.A.C. 